\title{
Rosacea and Oxidative Stress: Mini Review
}

\author{
Suzan Demir Pektas* \\ Department of Dermatology, Mugla Sitki Kocman University, Turkey
}

*Corresponding author: Suzan Demir Pektas, Department of Dermatology, Mugla Sitki Kocman University, Faculty of Medicine, 48000, Mugla, Turkey

Submission: 悳 June 13, 2018; Published: 眥 July 18, 2018

\begin{abstract}
Rosacea is a chronic inflammatory skin disorder. The average incidence is around $10 \%$. The prevalence of the disease is higher in females and ages between 20-50 years. Oxidative stress is an disequilibrium among oxidants and antioxidants. According to many of studies, reactive oxygen species along with decreased antioxidant levels are responsible for rosacea pathogenesis.
\end{abstract}

Keywords: Rosacea; Oxidative stress; Pathogenesis; Inflammatory dermatosis; Telangiectasia

Abbreviations: OS: Oxidative Stress; ROS: Reactive Oxygen Species; TLR2: Toll Like Receptor 2

\section{Introduction}

Rosacea is a long-standing is an inflammatory dermatosis characterized by flare-ups and remissions. Patients apply to the dermatologists with complaints such as telangiectasia, edema, erythema, acneiform eruptions [1,2]. Individuals aged $20-50$ years are more commonly affected and the disease is more prevalent in women than in men. The average incidence is around $10 \%[1,3]$. In the previous studies, rosacea is reported to be related to systemic diseases such as hyperlipidemia, hypertension, insulin resistance, metabolic and cardiovascular diseases [4-9]. Its etiology is exactly unknown. Factors involved in the inflammatory proceses are also responsible for the pathogenetic factors in the rosacea. Morever, oxidative stress affects both the onset and the progression of rocasea [1-3].

Oxidative Stress (OS) is an disequilibrium among oxidants and antioxidants. It causes a impairment of redox signaling and controlor molecular abnormality. Excess levels of Reactive Oxygen Species (ROS) may give irrevesible damage to various molecules such as protein, DNA and lipids. Increased IL-1 and TNF-a levels cause damage in keratinocytes, fibroblasts and endothelial cells resulting in the recruitment of leucocytes from periheral blood to the tissues [2]. In the recent studies, it has been found that production of ROS from neutrophils are related to inflammation in rosacea [1-5]. According to these studies, elevated ROS substances and ROS together with decreased antioxidant levels are responsible for rosacea pathogenesis [1-3,5,6]. Now-a-days, it has been discovered that levels of cathelicidin, trypsin-like serine protease kallikrein 5 (KLK5) and Toll Like Receptor 2 (TLR2) are high in rosacea patients $[7,8]$.
Rosacea is thought to be a cutaneous vascular disorder but inflammation also plays an important role in the pathogenesis of rosacea. This inflammation is related to vascular and oxidative tissue changes and sunlight damage $[2,4]$. Oxidative modulation of proteins and lipids, deactivation of natural defences by ROS, altered lipid balance and the ROS-related stimulation of inflammatory mediators compose the inflammation of rosacea [2,4]. Recent studies have shown that rosacea is associated with an increase in ROS production and OS markers and a decrease in antioxidant levels $[1-3,6,7]$. ROS levels were evaluated in skin biopsy samples from rosacea and healthy individuals, and higher ROS activity in rosacea skin lesions than healthy controls was observed [5]. A defective antioxidant system in rosacea patients reported by serum total antioxidative potential levels [1], serum paraoxonase and arylesterase activities [2], superoxide dismutase activities [3] and antioxidant potential levels [10]. In the study, rosacea patients were divided into three groups based on clinical severity: stage I, II, and III [3]. They found no significant differences between Malondialdehyde (MDA) levels and Superoxide Dismutase (SOD) activities of the control and stage I and stage II groups classified as mild forms of rosacea. On the otherhand,in the severe involvement group (stage III), the SOD activity was lower than in the control groupwith increased MDA levels.The authors hypothesised that in the mild involvement phase of rosacea patients, SOD activity was stimulated to protect the skin against ROS so that the MDA levels were maintained. In contrast, inmore severe disease form, due to a decrease in the capacity of the antioxidant defence system, the MDA levels were increased.Baz and colleagues [6] examinedplasma 
MDA, and antioxidant potential levels in rosacea patients and control subjects. They discovered higher MDA levels and lower antioxidant potential levels in rosacea when compared to the control group, regardless of the severity of the disease. A positive correlation was detected between MDA and antioxidant potential levels [6]. Tisma VS et al. [1] found signicantly higher levels of serum peroxide and significantly lower serum total antioxidative levelsin rosacea patients compared with the healthy subjects. We also found increased disulfide levels, and disulfide/native thiolratios in rosacea patients compared to healthy subjects, suggesting increased oxidative stress in rosacea, which was similar to the results of previous studies [1-3].

Since the subytpes of rosacea are clinically heterogeneous, rosacea studies were diversely conducted based on the findings in clinical manifestations, histology, and factors exacerbating the skin disorder. From the diverse findings, the complex network in the pathophysiology of all rosacea subytpesis still unclear and expected to be from multiple factors innate immunity, vascular changes, reactive oxygen species, ultra violet radiation, and microbes [1-3]. Regarding the role of oxidative stress in different types of rosacea patients, Takci $\mathrm{Z}$ et al. [2] reported decreased paraoxonase and arylesterase activities and increased serum lipid hydroperoxide levels suggestingelevated oxidative stress in patients with rosacea compared with controls, however, there were no significant differences in these parametersamong ERT, PPT and phymatoussubtype of the rosacea patients.

\section{Conclusion}

The pathophysiology of rosacea is multifactorial and in completely understood. It is reported that inflammation is a mainly process in rosacea. This inflammation is associated with many of factor including UV damage, vascular changes, and oxidative tissue damage. In order to determine role of OS on this disease or to understand the association between oxidative stress, inflammation and rocasea, further studies including large number of patients are needed.

\section{References}

1. Tisma VS, Basta Juzbasic A, Jaganjac M, Brcic L, Dobric I, et al. (2009) Oxidative stress and ferritin expression in the skin of patients with rosacea. J Am Acad Dermatol 60(2): 270-276.

2. Takci Z, Bilgili SG, Karadag AS, Kucukoglu ME, Selek S, et al. (2015) Decreased serum paraoxonase and arylesterase activities in patients with rosacea. J Eur Acad Dermatol Venereol 29(2): 367-370.

3. Oztas MO, Balk M, Ogus E, Bozkurt M, Ogus IH, et al. (2003) The role of free oxygen radicals in the aetiopathogenesis of rosacea. Clin Exp Dermatol 28(2): 188-192.

4. Akin Belli A, Ozbas Gok S, Akbaba G, Etgu F, Dogan G (2016) The relationship between rosacea and insulin resistance and metabolic syndrome. Eur J Dermatol 26(3): 260-264.

5. Rainer BM, Fischer AH, Luz Felipe da Silva D, Kang S, Chien A (2015) Rosacea is associated with chronic systemic diseases in a skin severitydependent manner: results of a case-control study. J Am Acad Dermatol 73(4): 604-608.

6. Egeberg A, Hansen PR, Gislason GH, Thyssen JP (2016) Clustering of autoimmune diseases in patients with rosacea. J Am Acad Dermatol 74(4): 667-672.

7. Duman N, Ersoy Evans S, Atakan N (2014) Rosacea and cardiovascular risk factors: a case control study. J Eur Acad Dermatol Venereol 28(9): 1165-1169.

8. Rainer BM, Fischer AH, Luz Felipe da Silva D, et al. (2015) Rosacea is associated with chronic systemic diseases in a skin severity dependent manner: results of a case-control study. J Am Acad Dermatol 73(4): 604608.

9. Hua TC, Chung PI, Chen YJ, Wu LC, Chen YD, et al. (2015) Cardiovascular comorbiditiesin patients with rosacea: a nationwide case-control study from Taiwan. J Am Acad Dermatol 73(2): 249-254.

10. Baz K, Cimen MY, Kokturk A, Aslan G, Ikizoglu G, et al. (2004) Plasma reactive oxygen species activity and antioxidant potential levels in rosacea patients: correlation with seropositivity to Helicobacter pylori. Int J Dermatol 43(7): 494-497.
Creative Commons Attribution 4.0 International License

For possible submissions Click Here

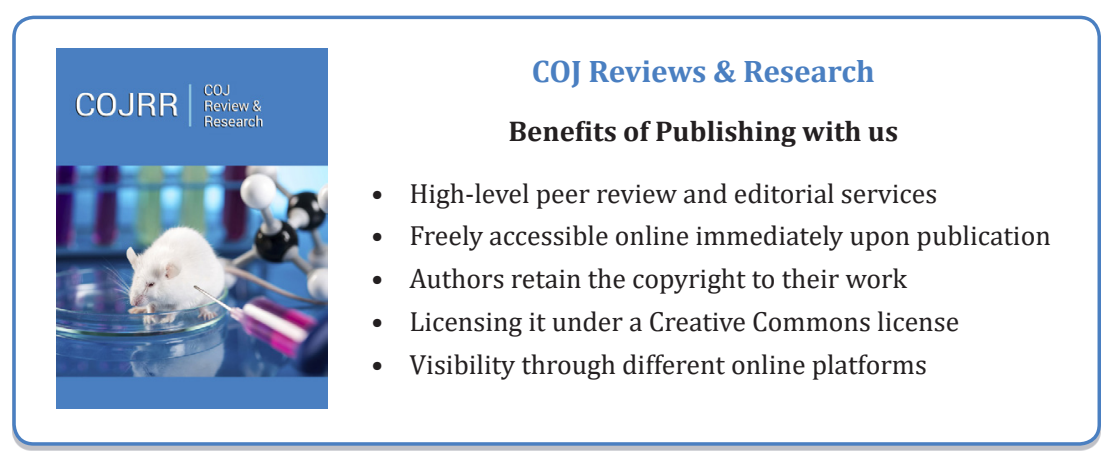

